\title{
Genome-wide SNP identification and QTL mapping for black rot resistance in cabbage
}

\author{
Jonghoon Lee ${ }^{1}$, Nur Kholilatul Izzah" ${ }^{1,2}$, Murukarthick Jayakodi', Sampath Perumal ${ }^{1}$, Ho Jun Joh', Hyeon Ju Lee ${ }^{1}$, \\ Sang-Choon Lee', Jee Young Park', Ki-Woung Yang ${ }^{1,3}$, II-Sup Nou ${ }^{3}$, Joodeok Seo ${ }^{4}$, Jaeheung Yoo ${ }^{4}$, \\ Youngdeok Suh ${ }^{4}$, Kyounggu Ahn ${ }^{4}$, Ji Hyun Lee ${ }^{5}$, Gyung Ja Choi ${ }^{5}$, Yeisoo Yu ${ }^{6}$, Heebal Kim ${ }^{7,8}$ and Tae-Jin Yang ${ }^{1 *}$
}

\begin{abstract}
Background: Black rot is a destructive bacterial disease causing large yield and quality losses in Brassica oleracea. To detect quantitative trait loci (QTL) for black rot resistance, we performed whole-genome resequencing of two cabbage parental lines and genome-wide SNP identification using the recently published B. oleracea genome sequences as reference.

Results: Approximately $11.5 \mathrm{~Gb}$ of sequencing data was produced from each parental line. Reference genome-guided mapping and SNP calling revealed 674,521 SNPs between the two cabbage lines, with an average of one SNP per 662.5 bp. Among 167 dCAPS markers derived from candidate SNPs, 117 (70.1\%) were validated as bona fide SNPs showing polymorphism between the parental lines. We then improved the resolution of a previous genetic map by adding 103 markers including 87 SNP-based dCAPS markers. The new map composed of 368 markers and covers $1467.3 \mathrm{cM}$ with an average interval of $3.88 \mathrm{cM}$ between adjacent markers. We evaluated black rot resistance in the mapping population in three independent inoculation tests using $F_{2: 3}$ progenies and identified one major QTL and three minor QTLS.

Conclusion: We report successful utilization of whole-genome resequencing for large-scale SNP identification and development of molecular markers for genetic map construction. In addition, we identified novel QTLs for black rot resistance. The high-density genetic map will promote QTL analysis for other important agricultural traits and marker-assisted breeding of $B$. oleracea.
\end{abstract}

Keywords: Cabbage, Whole-genome resequencing, Genetic linkage map, Black rot, QTL

\section{Background}

Cabbage (Brassica oleracea L.) is one of the most important vegetable crops, and is consumed as a food worldwide due to its healthy compounds for humans. Besides its economic importance, cabbage is considered a valuable plant for the study of genome evolution because it contains a CC genome, which represents one of three basic diploid Brassica species in the U's triangle [1]. Recently, two draft genome sequences of B. oleracea were reported $[2,3]$, and the availability of this reference genome enhances our understanding of the genome architecture of B. oleracea and the evolution of Brassica

\footnotetext{
* Correspondence: tjyang@snu.ac.kr

'Department of Plant Science, Plant Genomics and Breeding Institute, and Research Institute of Agriculture and Life Sciences, College of Agriculture and Life Sciences, Seoul National University, Seoul 151-921, Republic of Korea Full list of author information is available at the end of the article
}

species, as well as facilitates identification of genes associated with important traits for breeding.

Black rot is one of the most devastating diseases to crucifers including $B$. oleracea and is caused by the vascular bacterium Xanthomonas campestris pv. campestris (Pammel) Dowson $(X c c)$. The disease infects the host plants through hydathodes, wounded tissue, insects and stomata $[4,5]$. The main disease symptoms are $\mathrm{V}$-shaped chlorotic lesions at the margins of leaves, necrosis and darkening of leaf veins, which lead to serious production losses in vegetable crops [6]. Accordingly, development of cultivars resistant to black rot has been a priority for breeders.

Several methods have been attempted to control black rot disease, including crop diversification and rotation, production of disease-free seed, pre-treatment of seed with bactericide, elimination of potential pathogen sources 
such as infected crop debris and weeds, and planting of resistant cultivars [7]. Among these, utilization of resistant cultivars is one of the most effective and efficient ways to reduce disease incidence and crop loss. However, the development of commercially acceptable resistant varieties has proven to be extremely difficult due to the lack of studies on genetics and breeding for resistance in cabbage. Two major factors hinder black rot resistance breeding in B. oleracea: multigenic control of resistance and emergence of new races of the pathogen that overcome host resistance [8]. Nine races of $X c c$ have been identified [9], among which races 1 and 4 are the major pathogens causing black rot disease in B. oleracea crops [10]. Therefore, obtaining $B$. oleracea cultivars that have resistance to both races is considered a prerequisite to control black rot disease [11].

Molecular markers are highly useful for genomic analysis and allow exploration of heritable traits and the corresponding genomic variation [12]. DNA markers are now key components of crop improvement programs, and are applied to identify cultivars, analyze genetic diversity, construct linkage maps and identify quantitative trait loci (QTL) [13]. Advances in molecular markers have facilitated the identification of interesting traits via marker-assisted selection (MAS) in plant improvement. Marker-based approaches represent an effective and rapid strategy for identifying and transferring useful genes in breeding programs [14]. Furthermore, the identification of markers linked to QTL can allow analysis of the consistency of QTL effects across different environments and genetic backgrounds, and increase the frequency of favorable alleles during selection [15]. Several QTLs for black rot resistance in B. oleracea have been reported, including two on linkage groups 1 and 9, and two additional QTLs on linkage group 2 [15], as well as two other major QTLs on linkage groups 2 and 9, and two minor QTLs on linkage groups 3 and 7 [16]. Moreover, three QTLs analyzed using SNP markers in the $\mathrm{F}_{2}$ mapping population derived from a cross between resistant cabbage and susceptible broccoli were found on linkage groups 2, 4 and 5, and exhibited significant effects in black rot resistance [4]. Recently, three further QTLs for black rot resistance were also detected in linkage groups 5, 8 and 9 [5]. In total, 14 QTLs with major and minor effects have been mapped on eight different $B$. oleracea chromosomes, suggesting that resistance to black rot disease is complex and quantitatively controlled by multiple genes in $B$. oleracea.

Successful QTL mapping requires a large number of genetic markers [17]. Markers based on simple sequence repeats (SSRs) and single nucleotide polymorphisms (SNPs) are commonly used due to their advantages over other types of genetic markers. SSR markers are highly reproducible, highly polymorphic, and amenable to automation. However, next-generation sequencing (NGS) technology makes SNP markers preferable to SSR markers [18]. SNPs have proved to be universal as well as the most abundant forms of genetic variation even among individuals of the same species [19]. Therefore, SNP markers exhibit higher polymorphism than SSR markers [20,21].

In this study, we have resequenced two parental cabbage lines up to $20 \times$ genome coverage and conducted a genome-wide survey for SNPs. We validated the SNPs and developed derived cleaved amplified polymorphic sequences (dCAPS) markers for resistance against black rot disease. The genome-wide catalog of SNPs, the high-density map derived from a mapping population generated from elite cabbage breeding lines with a narrow genetic background, and the QTLs reported herein all will be valuable for both breeding and genetic research in B. oleracea.

\section{Results}

Whole-genome resequencing of two cabbage parental lines and SNP detection

Whole genome sequencing data included about 114 million raw reads for $\mathrm{C} 1184$ and 113 million for C1234 (Table 1). The recently assembled $B$. oleracea genome sequence consists of $488.6 \mathrm{Mb}$, including $446.9 \mathrm{Mb}$ in 9 pseudo-chromosomes and $41.2 \mathrm{Mb}$ of unanchored scaffolds, and corresponding to almost $75 \%$ of the estimated genome size $(648 \mathrm{Mb})$ [3]. Our new sequencing data represented approximately 18 -fold genome coverage for both parental lines based on the estimated genome size. We mapped each set of paired reads onto the nine pseudo-chromosomes of reference genome sequence. In total, almost 94 million raw reads (82.1\%) and 88 million (77.6\%) from C1184 and C1234, respectively, were successfully aligned to the reference genome. The average mapping depth was 21.2- and 20-fold for $\mathrm{C} 1184$ and $\mathrm{C} 1234$, respectively.

The total number of SNPs relative to the reference sequence and average SNP densities were very similar in both parental lines. Approximately 1.20 and 1.24 million high-quality SNPs are identified in C1184 and C1234,

Table 1 Summary of whole-genome resequencing data for B. oleracea lines

\begin{tabular}{lll}
\hline & $\mathrm{C} 1184$ & $\mathrm{C} 1234$ \\
\hline Raw reads & $114,454,524$ & $113,830,992$ \\
Raw bases & $11,559,906,924$ & $11,496,930,192$ \\
Coverage of B.oleracea genome & $17.8 \times$ & $17.7 \times$ \\
GC (\%) & 36.1 & 35.6 \\
Mapped reads & $93,956,750$ & $88,382,752$ \\
Mapped percentage (\%) & 82.1 & 77.6 \\
Mapped bases & $9,489,631,750$ & $8,926,657,952$ \\
Mapping depth (average) & 21.2 & 20.0 \\
\hline
\end{tabular}


respectively, by comparison to the reference genome. On average, a SNP was detected in each $372.8 \mathrm{bp}$ in C1184, and each 360.0 bp in C1234. Chromosome C03 of both lines had the most SNPs, whereas the fewest SNPs were found on chromosome C06 of C1184 and chromosome C04 of C1234.

These SNPs were merged and used to detect SNPs between the two parental lines (Table 2). As a result, a total of 674,521 SNPs were found throughout nine chromosomes, with an average of 1 SNP per 662.5-bp interval. The highest density of SNPs was found on chromosome C03, with a SNP per $541 \mathrm{bp}$, while the lowest density was on chromosome C05, with one SNP per $818.9 \mathrm{bp}$. Analysis of the distribution of SNPs per $100 \mathrm{~kb}$ along the nine chromosomes revealed areas of high and low SNP density on each chromosome (Figure 1).

\section{Development of dCAPS markers and construction of genetic map}

We used the SNPs between C1184 and C1234 for development of dCAPS markers. Based on the physical positions of all markers used in a previous genetic map for B. oleracea [21], new dCAPS markers were designed for the regions of low marker density. Among 167 markers amplified, 117 (70.1\%) were polymorphic between the two parental lines (Table 2 and Additional file 1: Table S1). Among the 117 polymorphic markers, 26 showed heterozygosity in one of parental lines (Table 2). We used 87 of these polymorphic dCAPS markers for genotyping of each individual in the $\mathrm{F}_{2}$ population (Additional file 1: Table S1). Additionally, 16 other types of polymorphic markers including five EST-based dCAPS markers, five MIP markers, three IBP markers, two genomic SSR markers, and one INDEL marker were also genotyped with the same population. Among 103 newly analyzed markers, 25 markers showed a segregation pattern distorted from the 1:2:1 Mendelian ratio in the $F_{2}$ population, based on chi-square goodness of fit at the 0.05 probability level (Additional file 2: Table S2). There were six segregation distortion regions (SDRs) in the previous map [21], and all dCAPS markers designed from the SDRs of C01 and C05 showed the same distortion ratio.

The 103 novel polymorphic marker loci (Additional files 1 and 2: Tables S1 and S2) were added to the previous 265 markers [21] to develop a higher density genetic map. All 368 markers were placed on the map, and a linkage map was generated with nine linkage groups (LGs) in which each LG had more than 32 markers (Figure 2, Table 3). The improved B. oleracea genetic map spanned 1,467.3 cM, which is $135.4 \mathrm{cM}$ more than the previous map, and the average distance between neighboring loci was reduced to 3.88 from $5.02 \mathrm{cM}$. Most of the new dCAPS markers were mapped to the originally estimated position of each chromosome sequence. The exceptions included BoRSdcaps1-35, which was designed on chromosome C01 but mapped to chromosome C02, and BoRSdcaps5-18, designed on chromosome C05 but mapped to chromosome $\mathrm{C} 09$.

\section{Black rot resistance assays and QTL analysis}

We performed three independent inoculation trials over three years. The final disease index for $F_{2}$ plants was determined by calculating the average value of the black rot disease indices for $10 \sim 15 \mathrm{~F}_{2: 3}$ progeny plants for each trial. Although all three inoculation tests were performed under the same conditions, the disease symptoms for each test were not consistent and tended to become more severe in later years (Additional file 3: Figure S1), possibly due to differences in plant growth or storage term for the $F_{3}$ seeds or to weather differences between years.

Table 2 Summary of SNPs detected from B. oleracea whole-genome resequencing data and development of dCAPS markers for validation

\begin{tabular}{|c|c|c|c|c|c|c|c|c|c|}
\hline \multirow{3}{*}{$\begin{array}{l}\text { Ch. } \\
\mathrm{C01}\end{array}$} & \multicolumn{6}{|c|}{ Number of SNPs (average bp per SNP) } & \multicolumn{3}{|l|}{ Validation } \\
\hline & \multicolumn{2}{|c|}{ Ref vs. C1184 } & \multicolumn{2}{|c|}{ Ref vs. C1234 } & \multicolumn{2}{|c|}{ C1184 vs. C1234 } & \multirow{2}{*}{$\begin{array}{l}\text { Amplified/Designed } \\
31 / 35\end{array}$} & \multirow{2}{*}{$\begin{array}{l}{\text { Polymorphic }(h)^{a}}^{20(4)}\end{array}$} & \multirow{2}{*}{$\begin{array}{l}\%^{b} \\
64.5 \%\end{array}$} \\
\hline & 122,191 & $(358.2)$ & 114,778 & $(381.3)$ & 66,197 & $(661.1)$ & & & \\
\hline $\mathrm{CO2}$ & 149,730 & (353.2) & 161,246 & $(328.0)$ & 74,741 & (707.6) & $14 / 17$ & $10(1)$ & $71.4 \%$ \\
\hline $\mathrm{CO3}$ & 196,150 & $(331.3)$ & 205,306 & $(316.5)$ & 120,115 & $(541.0)$ & $13 / 22$ & $11(1)$ & $84.6 \%$ \\
\hline $\mathrm{CO4}$ & 136,815 & $(392.6)$ & 132,144 & $(406.5)$ & 86,999 & (617.5) & $14 / 20$ & $8(4)$ & $57.1 \%$ \\
\hline $\mathrm{CO5}$ & 130,557 & $(359.2)$ & 132,887 & $(353.0)$ & 57,417 & (818.9) & $15 / 18$ & $14(2)$ & $93.3 \%$ \\
\hline $\mathrm{C06}$ & 87,712 & $(454.1)$ & 102,422 & (388.8) & 63,017 & (631.9) & $28 / 34$ & $16(4)$ & $57.1 \%$ \\
\hline $\mathrm{CO7}$ & 119,275 & $(405.5)$ & 128,978 & $(375.0)$ & 69,905 & (691.9) & $8 / 15$ & $5(1)$ & $62.5 \%$ \\
\hline $\mathrm{CO8}$ & 108,586 & (384.6) & 113,956 & (366.4) & 68,361 & (610.9) & $21 / 26$ & $18(3)$ & $85.7 \%$ \\
\hline C09 & 147,866 & (369.8) & 149,581 & (365.6) & 67,768 & (806.9) & $23 / 35$ & $15(6)$ & $65.2 \%$ \\
\hline Total & $1,198,882$ & (372.8) & $1,241,298$ & $(360.0)$ & 674,521 & (662.5) & $167 / 222$ & $117(26)$ & $70.1 \%$ \\
\hline
\end{tabular}

${ }^{a} \mathrm{~h}$ is the number of markers that showed heterozygous results.

${ }^{b}$ Percentage of the total amplified dCAPS markers that were polymorphic. 


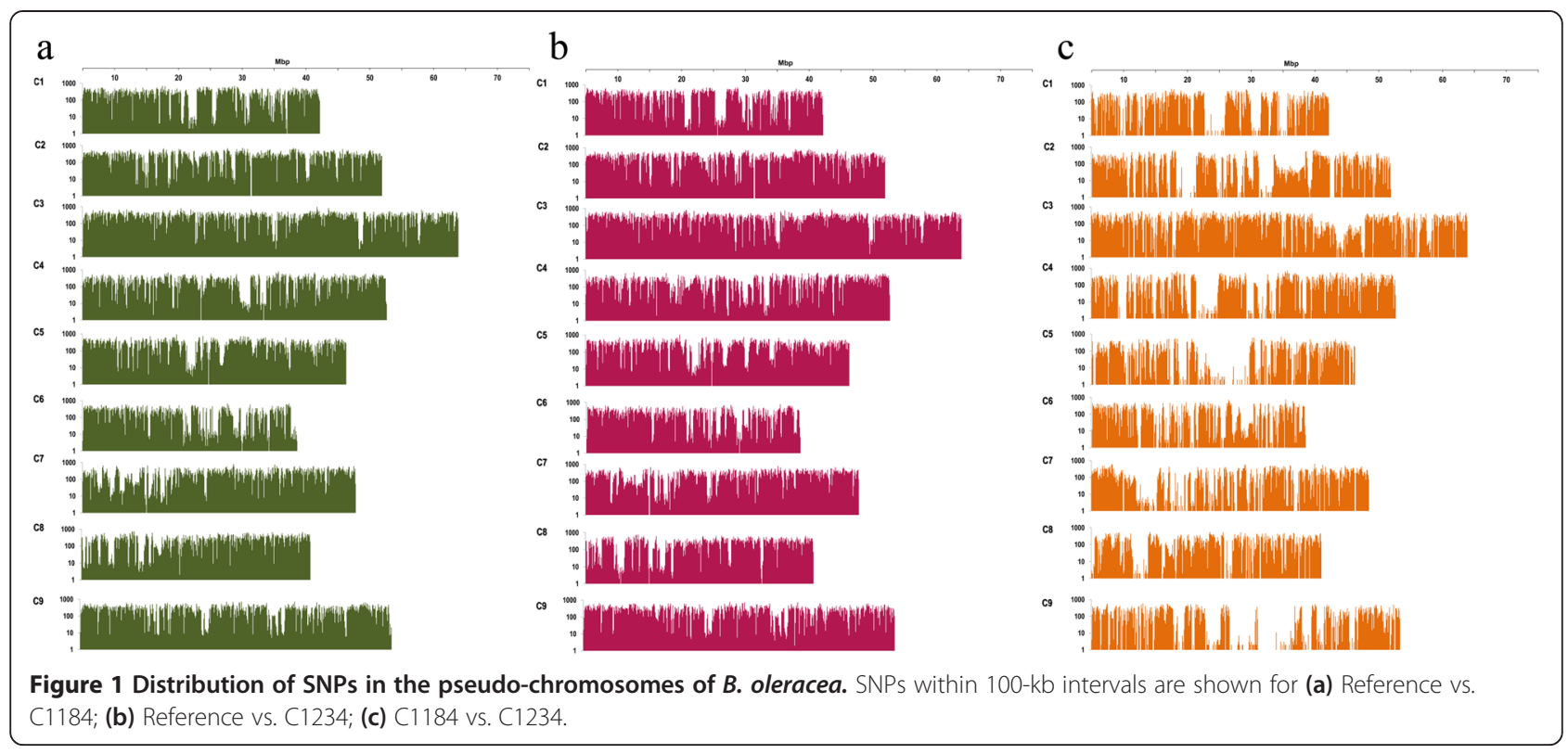

QTL analyses were performed for each of three trials. We detected significant QTLs, based on higher LOD scores than the thresholds calculated in the permutation tests; LOD threshold values for the tests in 2012, 2013, and 2014 were $3.063,2.912$, and 2.906 , respectively. In the first test performed in 2012, there were three significant QTL regions: BRQTL-C1_1 and BRQTL-C1_2 on chromosome C01, and BRQTL-C3 on chromosome C03 (Figure 2). Among these, BRQTL-C1_2 had the highest LOD score, additive effect, and variance explained (Table 4, Figure 2). The second test identified only a single QTL, which was included within BRQTL-C1_2 detected in the 2012 test, although this QTL had smallest LOD score among all QTLs identified in the three tests. The last test, carried out in 2014, identified BRQTL-C1_1 and 2 as well as a novel QTL in chromosome 6, BRQTL-C6. BRQTL-C1_2 in the 2014 test was identified as a smaller region than in 2012, but had the highest LOD score among all QTLs and accounted for $27.3 \%$ of the variation.

\section{NBS-encoding genes in QTL regions}

In most plants, disease resistance-related genes ( $R$ genes) encode proteins containing nucleotide binding sites (NBS) and a series of leucine-rich repeats (LRRs), termed NBS-LRR proteins. NBS-LRR proteins recognize and correspond to pathogen avirulence factors, and lead to defense responses and hypersensitive reactions [22]. Hence, we compared our genetic map to the pseudochromosome sequences [3] and searched for NBS-LRR genes within the QTL regions (Table 5). BRQTL-C1_1 was found between markers H073E22-3 and BoRSdcaps1-11, and BRQTL-C1_2 was between BoRSdcaps1-13 and BoEdcaps4 (Table 4). We identified eight NBS-LRR- encoding genes between H073E22-3 and BoEdcaps4 showing BRQTL-C1_1 and BRQTL-C1_2 QTLs. Seven NBS-LRR type $\mathrm{R}$ genes were detected within $1 \mathrm{Mb}$ of the BoESSR291 marker, which is located near the BRQTL-C3 region. BRQTL-C6 contained five NBS-LRR type R genes.

We compared the sequences of these 21 candidate $R$ genes against the Brassica Database (BRAD; http://brassicadb.org/) [23]. All 21 sequences showed similarity to disease resistance proteins, of which 19 and 11 sequences had syntenic genes in $B$. rapa and $A$. thaliana, respectively. According to the gene annotation, two candidate disease resistance genes (Bo1g094680 and Bo1g094710) in BRQTL-C1, and seven genes in BRQTL-C3 were found as gene clusters (Table 5).

Seven of nine NBS-LRR genes in BRQTL-C1 were syntenic with the $\mathrm{R}$ genes in the counterpart regions of chromosome A01 in B. rapa (Table 5). Orthologous genes of two NBS-LRR genes, Bo1g094680 and Bo1G094710, located within a $63-\mathrm{Kb}$ portion of BRQTL-C1 appeared as tandem array at the counterpart syntenic region in $B$. rapa and $A$. thaliana (Figure 3a). All NBS-LRR genes in BRQTL-C6 also showed highly conserved syntenic relationships with counterpart regions in B. rapa and A. thaliana. However, a 72-Kb region near BRQTL-C3 contained a cluster of seven NBS-LRR genes, whereas the syntenic region in $B$. rapa contained a cluster of only three such genes, and the corresponding syntenic region in A. thaliana did not have any $\mathrm{R}$ genes (Figure $3 \mathrm{~b}$ ).

\section{Discussion}

Frequency and utility of SNPs revealed by whole-genome resequencing

An appropriate reference sequence allows whole-genome sequence data from individuals to be aligned, and thus 


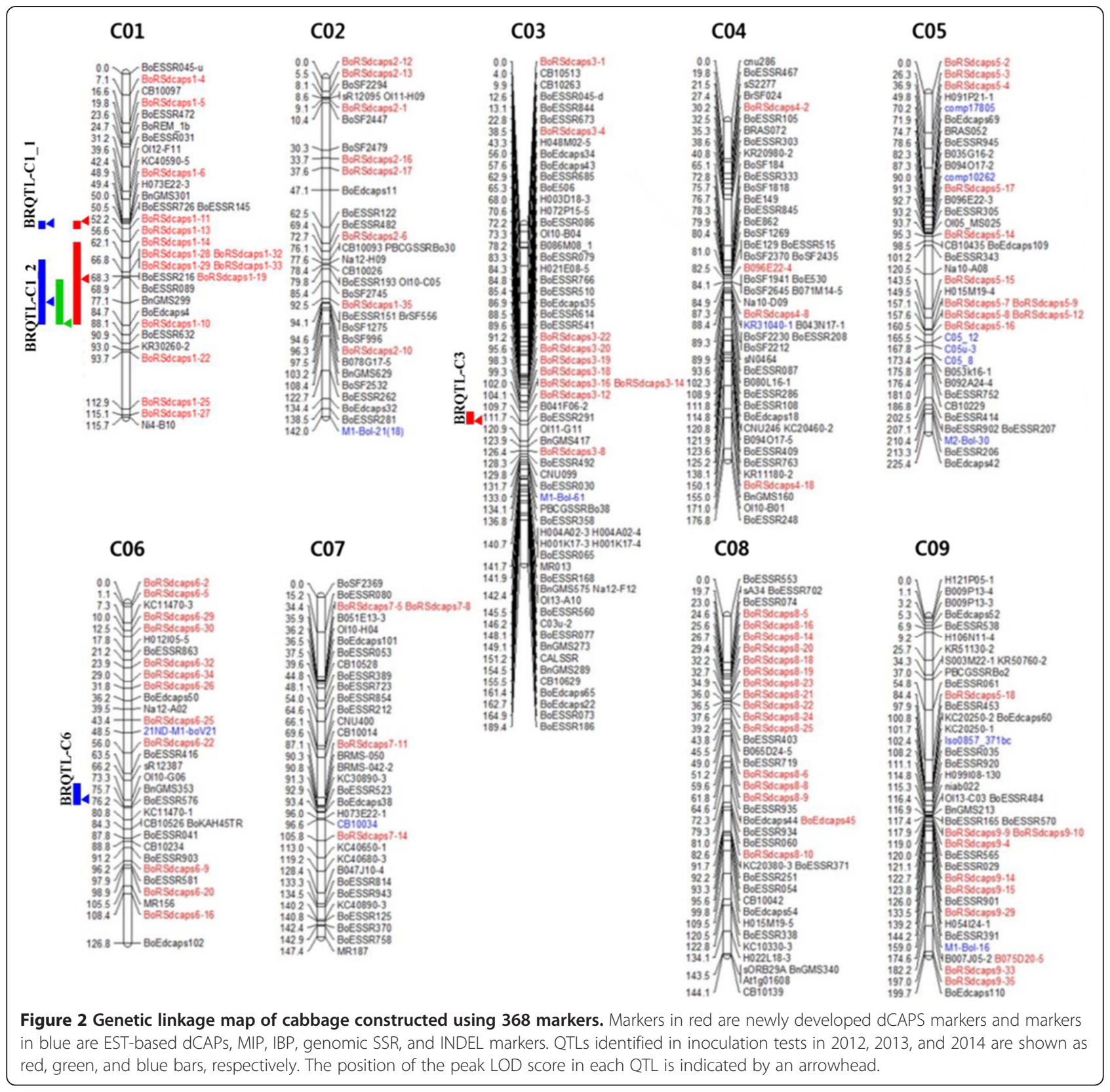

Table 3 Distribution of molecular markers on the cabbage genetic map

\begin{tabular}{llllllllllll}
\hline Marker type & & C01 & C02 & C03 & C04 & C05 & C06 & C07 & C08 & C09 & Total \\
\hline This study & dCAPS & 15 & 8 & 10 & 3 & 11 & 12 & 4 & 15 & 9 & 87 \\
& Other markers $^{\mathrm{a}}$ & 0 & 1 & 1 & 2 & 6 & 1 & 1 & 1 & 3 & 16 \\
Previous study & 18 & 25 & 52 & 43 & 23 & 19 & 29 & 25 & 31 & 265 \\
Total & 33 & 34 & 63 & 48 & 40 & 32 & 34 & 41 & 43 & 368 \\
Length (cM) & 115.7 & 142.0 & 189.4 & 176.8 & 225.4 & 126.8 & 147.4 & 144.1 & 199.7 & 1467.3 \\
Average interval (cM) & 3.51 & 4.18 & 3.01 & 3.68 & 5.64 & 3.96 & 4.34 & 3.51 & 4.64 & 3.88
\end{tabular}

Including MITE insertion polymorphism, EST-based dCAPS, Intron based polymorphism, genomic SSR, and INDEL markers. 
Table 4 QTLs identified for resistance to Xcc KACC 10377

\begin{tabular}{|c|c|c|c|c|c|c|c|}
\hline $\begin{array}{l}\text { Inoculation } \\
\text { test }\end{array}$ & QTL name & $\begin{array}{l}\text { Linkage } \\
\text { group }\end{array}$ & Marker interval (cM) & $\begin{array}{l}\text { Marker nearest to } \\
\text { peak in LOD score }\end{array}$ & $\operatorname{LOD}^{a}$ & $\begin{array}{l}\text { Additive } \\
\text { effect }^{\mathrm{b}}\end{array}$ & $\begin{array}{l}\text { Variance explained } \\
(\%)^{c}\end{array}$ \\
\hline \multirow[t]{3}{*}{ 1st test (2012) } & BRQTL-C1_1 & C1 & H073E22-3 - BoRSdcaps1-11 (2.8 cM) & BnGMS301 & 3.871 & -0.714 & 17.8 \\
\hline & BRQTL-C1_2 & $\mathrm{C} 1$ & BoRSdcaps1-13 - BoEdcaps4 (28.1 cM) & BoESSR089 & 4.720 & -0.697 & 21.2 \\
\hline & BRQTL-C3 & $\mathrm{C} 3$ & BoRSdcaps3-12 - BoESSR291 (7.6 cM) & B041F06-2 & 3.834 & -0.661 & 17.6 \\
\hline 2nd test (2013) & BRQTL-C1_2 & $\mathrm{C} 1$ & BoESSR089 - BoEdcaps4 (15.8 cM) & BoEdcaps4 & 3.051 & -0.602 & 15.1 \\
\hline \multirow[t]{3}{*}{ 3rd test (2014) } & BRQTL-C1_1 & $\mathrm{C} 1$ & H073E22-3 - BoRSdcaps1-11 (2.8 cM) & $\begin{array}{l}\text { BoESSR726, } \\
\text { BoESSR145 }\end{array}$ & 3.881 & -0.912 & 19.8 \\
\hline & BRQTL-C1_2 & $\mathrm{C} 1$ & BoRSdcaps1-14 - BoEdcaps4 (22.6 cM) & BnGMS299 & 5.619 & -0.987 & 27.3 \\
\hline & BRQTL-C6 & $\mathrm{C} 6$ & sR12387 - BnGMS353 (9.5 cM) & Ol10-G06 & 3.847 & -0.868 & 19.6 \\
\hline
\end{tabular}

Shown are position of the QTL on the map, LOD scores, additive and dominant effects, and percentage of variance explained.

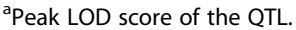

${ }^{\mathrm{b}}$ Additive or dominant effect of $\mathrm{C} 1234$ allele.

'Percentage of variance explained at the peak of QTL.

resequencing can be used to detect genetic variation between different samples as high-confidence sequence differences [24]. Accordingly, we performed wholegenome resequencing for two cabbage lines to detect genome-wide SNPs for marker development to construct a high-density genetic map. There are two available draft genome sequences for $B$. oleracea; although the total assembled sequence of Liu et al. (539.9 Mb) [2] is larger than that of Parkin et al. (488.6 Mb) [3], the size of the nine pseudo-chromosomes of the latter $(446.9 \mathrm{Mb})$ is larger than that of the former $(388.8 \mathrm{Mb})$. Therefore, we chose the genome sequence of Parkin et al. [3] as a reference for this research because of its advantage for sequence-guided SNP marker development.
Approximately $80 \%$ of our newly generated PE sequence reads were successfully aligned to the reference genome. Almost 1.2 million SNPs were found in both lines compared to the reference, and 670,000 SNPs were found between $\mathrm{C} 1184$ and $\mathrm{C} 1234$. The number and density of SNPs between both parental lines were much lower than those detected by comparison with the reference. This could be related to the fact that the plant material used for the reference genome sequencing was kale-like B. oleracea [3], whereas the two parental lines used in this work were typical cabbages. In addition, we detected fewer SNPs between these lines than the 1.42 million SNPs (averaging one SNP in every $360 \mathrm{bp}$ ) previously reported between

Table 5 NBS-LRR-encoding genes in black rot resistance QTL regions identified for B. oleracea in this study, and syntenic orthologs in closely related species

\begin{tabular}{|c|c|c|c|c|}
\hline \multirow[t]{2}{*}{ QTL region in $B$. oleracea } & \multirow{2}{*}{$\begin{array}{l}\text { Genes in B. oleracea } \\
\text { (Parkin et al. } 2014 \text { [14]) }\end{array}$} & \multicolumn{2}{|l|}{ Orthologs in B. rapa } & \multirow{2}{*}{$\begin{array}{l}\text { Syntenic genes } \\
\text { in A. thaliana }\end{array}$} \\
\hline & & Gene ID ${ }^{b}$ & Position in B. rapa & \\
\hline$\overline{B R Q T L-C 1 \_1 ~}$ & Bo1g056920 & Bra034079 & A01: $25,091,903-25,095,843$ & \\
\hline \multicolumn{5}{|l|}{ C01: $14,884,502-16,579,946$} \\
\hline BRQTL-C1_2 & Bo1g057060/070 & Bra039560 & A01: 11,678,267 - 11,687,802 & AT4G14380 \\
\hline \multicolumn{5}{|l|}{ C01: $18,227,386-37,119,290$} \\
\hline & Bo1g086130 & Bra013691 & A01: 7,172,559- 7,175,366 & AT4G23440 \\
\hline & Bo1g087610 & Bra038144 & Scaffold 000140 & AT1G57850 \\
\hline & Bo1g091560 & & & \\
\hline & Bo1g094680/710 & Bra031456/455 ${ }^{\mathrm{a}}$ & A01: $17,128,737-17,140,522$ & AT1G61100/105 \\
\hline & Bo1g103860 & & & \\
\hline BRQTL-C3 & \multirow{2}{*}{$\begin{array}{l}\text { Bo3g060060/070/080/ } \\
100 / 110 / 130 / 140^{\mathrm{a}}\end{array}$} & \multirow[t]{2}{*}{ Bra001160/161/162 ${ }^{\mathrm{a}}$} & \multirow[t]{2}{*}{ A03: $15,040,407-15,054,981$} & \\
\hline C03: $19,714,632-22,846,644$ & & & & \\
\hline BRQTL-C6 & Bo6g025490 & Bra004192 & A07: $20,618,348-20,627,341$ & AT1G66840 \\
\hline C06: 7,423,787 - 10,466,894 & Bo6g031330/350/360/380 & Bra003997 & A07: $19,462,054-19,467,133$ & AT1G69550 \\
\hline
\end{tabular}

aTandemly arrayed genes.

${ }^{\mathrm{b}}$ Gene ID in BRAD database (http://brassicadb.org/). 


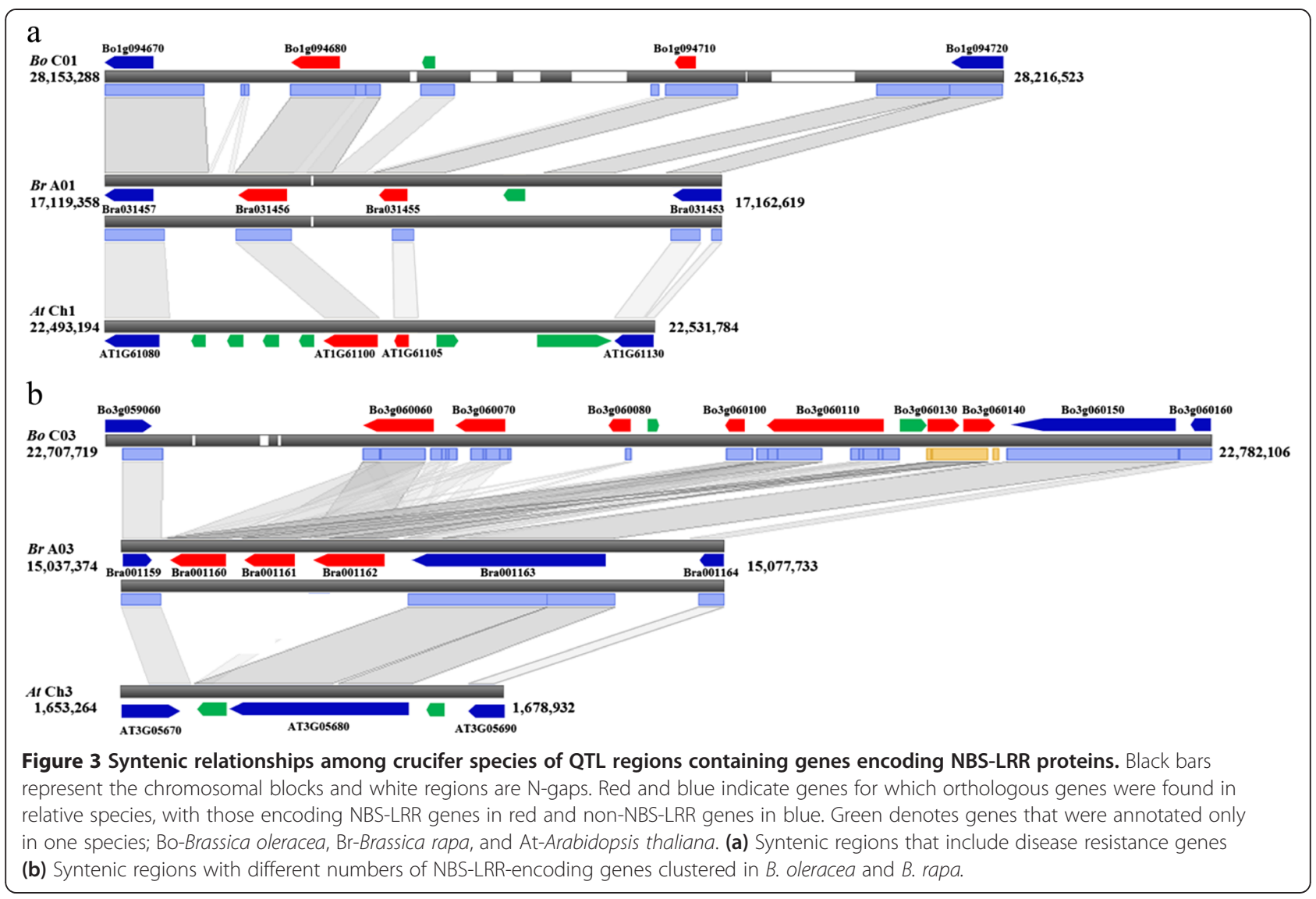

two other cabbages [25]. Our plant materials have been used as elite breeding resources by a Korean company, and thus the genetic relationship between these two parental inbred lines is likely much closer than to the reference accession or the relationship between the two cabbages used by Liu et al. [25]; this close relationship likely underlies the relatively low number of SNPs we identified.

Among 167 dCAPS markers that successfully produced PCR products, $70.1 \%$ showed polymorphism. This rate was much higher than that of EST-derived dCAPS markers, in which the polymorphic rate was $58.44 \%$ when evaluated with low-sequencing depth 454 RNA-seq reads [21]. Paired-end read data generated by Illumina sequencing could allow more accurate alignment of raw reads compared to single reads from 454 RNA-seq, and thus the SNP calling process would also become more precise. The $29.9 \%$ of dCAPS markers showing no polymorphism might reflect false mapping of reads to paralogous regions, as there is high sequence similarity between the triplicated genomes and among recently duplicated chromosome segments [26-28]. This could also be the reason two dCAPS markers, BoRSdcaps1-35 and BoRSdcaps5-18, were mapped to unexpected chromosomes.
Collectively, our results demonstrate that whole-genome resequencing data generated by NGS techniques can be highly useful for large-scale discovery of SNPs and development of SNP-based molecular markers. Further study will enable high-throughput genotyping with SNPs detected here.

\section{Improvement of the genetic map between cabbage breeding lines}

By obtaining large numbers of reliable SNPs and utilizing them for development of DNA markers, we were able to improve the genetic map of cabbage. The genetic map now spans a total 1,467.3 cM after our addition of SNP markers developed for the relatively large gaps (greater than $20 \mathrm{cM}$ ) in the previous map [21]. Consequently, the 12 gaps in the previous genetic map are now reduced to 6 gaps and the average interval is smaller than before. The 368 markers used for the improved genetic map are promising for general cabbage breeding purposes because the map was built using a mapping population between two elite breeding lines with narrow genetic diversity. By contrast, most of previous genetic map was built using mapping populations derived between lines with 
wide genetic diversity for academic purposes, for example a cross between double-haploid (DH) lines derived from other subspecies [3,29] or DH lines selected based on simple morphological differences [20]. Therefore, the genetic map in this study will be helpful for molecular breeding associated not only with black rot resistance but also with many other important agricultural traits.

\section{QTL mapping of black rot resistance}

We identified four QTL regions that could contribute additively to resistance. The BRQTL-C1_2 QTL region was detected repeatedly in the three independent inoculation tests, had the highest LOD values and also accounted for the highest percentage of the variation in all tests. Accordingly, BRQTL-C1_2 is a strong candidate to be a major QTL for black rot resistance. BRQTL-C1_1, BRQTL-C3, and BRQTL-C6 seem to be minor QTLs, which could be influenced by plant conditions and environmental factors. Although two QTLs identified in chromosome $\mathrm{C} 1$ included SDRs, we retained all distorted markers for QTL analysis because distorted markers can also be helpful for QTL mapping when they are addressed properly [30].

The positions of our black rot resistance QTLs did not coincide with those of the 14 previously reported QTLs $[4,5,15,16]$. This lack of overlap is probably due to differences in disease resistance sources or inocula used. Some studies did not describe the races used $[15,16]$, while some $[4,5]$ used $X c c$ race 1 . The exact race used in this study has not been classified yet. Integrated and standardized protocols for black rot disease races and testing would facilitate further research. However, even though the same $X c c$ race was used in our three inoculation tests, disease indices for same $F_{2}$ lineages were not consistent year to year and thus different QTLs were detected between tests. Resistance to Xcc has been reported to vary depending on accessions of B. oleracea and pathogen races [31,32]. Further, the resistance is likely also affected by complex polygenic control under different environmental conditions. Regardless of the race used (if the same as in previous studies or not), the different QTLs detected here should represent new regions.

\section{Candidate genes for black rot resistance}

The genomes of B. oleracea, B. rapa, and A. thaliana share a set of 24 conserved syntenic blocks, A to X, that can be identified among the ancestral karyotype [33]. The complete $B$. oleracea draft genome also demonstrates generally strong conservation with $B$. rapa in large segments at the pseudo-molecule level [2,3]. Comparative analysis revealed the presence of conserved $R$ gene orthologs at the syntenic counterparts in B. oleracea, B. rapa and
A. thaliana. In particular, the BRQTL-C1 region of $\mathrm{C} 1$ in $B$. oleracea showed large-scale conservation with A01 in B. rapa. Our analysis demonstrated that Bra038144, found in unanchored scaffold000140 of the $B$. rapa genome, is an ortholog of Bolg087610 in $B$. oleracea (Table 5). Based on our finding that AT1G57850, the corresponding orthologous gene in $A$. thaliana, was also located in a syntenic region, the unanchored B. rapa scaffold000140 is likely derived from chromosome A01.

In plant genomes, hundreds of NBS-LRR genes are distributed as single genes or in tandem arrays as gene clusters, which arise from tandem gene duplications or homologous recombination and homogenization $[34,35]$. We detected $21 \mathrm{R}$ genes in the four QTL regions, of which 9 were in gene clusters (Table 5). Most of the $\mathrm{R}$ genes showed conserved syntenic relationships in Brassica and Arabidopsis (Figure 3a). However, near BRQTL-C3 were NBS-LRR gene clusters that appear to be unique to the Brassica lineage (Figure $3 \mathrm{~b}$ ). This result implied that three-R-gene clusters arose by insertion in the Brassica lineage at BrA03 and subsequently amplified to a seven-R-gene cluster in B. oleracea over the 4.6 million years after divergence of the Brassica species [2].

Although genomes of Brassica-lineage species underwent whole-genome triplication events, the number of resistance genes was not proportionally increased in the Brassica genome [27,36]. Around $150 \sim 200 \mathrm{R}$ genes were reported in the A. thaliana genome [2,35,37], and 206 [2] 244 (http://brassicadb.org/) and 157 genes [2] were annotated as R genes in B. rapa and B. oleracea genomes, respectively. The 21 NBS-LRR genes found in the four QTL regions are proportionally higher density compared to other chromosomal regions, supporting the idea that some of these NBS-LRRs could be candidate to control black rot resistance in B. oleracea. Further analysis to reveal the function of these genes will be necessary for identification of the major resistance genes for Xcc.

\section{Conclusion}

We performed whole-genome resequencing of two cabbage inbred lines that are parental lines for black rot disease resistance and breeding lines with elite agricultural traits. Based on genome-wide SNP detection and validation with dCAPS markers, we report 670,000 SNPs with $70 \%$ accuracy between the parental lines. By combining SNP-based markers into the previous genetic map, we improved the genetic map and identified four QTL regions that contained 21 candidate $\mathrm{R}$ genes. We thus demonstrated that whole-genome resequencing can successfully be applied for detection of large-scale SNPs, development of molecular markers, and ultimately construction of a highdensity genetic map for QTL analysis and marker-assisted breeding of $B$. oleracea. 


\section{Methods}

\section{Plant materials and whole-genome resequencing}

Two cabbage (Brassica oleracea L. var. capitata) inbred lines, C1184 and C1234, were selected as parents to develop a mapping population. The two lines show different responses to black rot disease; $\mathrm{C} 1184$ is susceptible to $X$. campestris pv. campestris (Xcc), whereas C1234 is resistant. The mapping population consisted of $97 \mathrm{~F}_{2}$ plants generated by crossing between C1184 and C1234, as described previously [21]. Furthermore, the $97 \mathrm{~F}_{2}$ plants were vernalized and self-pollinated to produce seeds of $F_{3}$ progenies for inoculation tests. All plant materials examined in this study were obtained from Joeun Seeds Co. (Chungcheongbuk-Do, Korea).

Genomic DNAs were extracted from approximately $5 \mathrm{~g}$ samples of young leaves from the cabbage parental lines, following the modified cetyltrimethylammonium bromide (CTAB) protocol [38]. The quality and quantity of the DNA were examined using a NanoDrop ND-1000 (NanoDrop Technologies, Inc., USA). More than $5 \mu \mathrm{g}$ extracted DNA was randomly sheared and quantified using DNA 1000 kit (Agilent Technologies, Inc., USA) according to the manufacturer's protocol. Sequencing with constructed shotgun libraries of C1184 and C1234 was performed by Illumina Hi-seq 2000. Fragmentation, library construction, and sequencing were carried out by the National Instrumentation Center for Environmental Management (NICEM; Seoul, Korea).

\section{SNP discovery and dCAPS marker design}

Overall process of SNP discovery was performed by following the framework described by DePristo et al. [39]. Briefly, Illumina paired reads from the parental lines were aligned to the reference sequence of B. oleracea [3] using Bowtie2 program [40]. Then, read grouping and removal of PCR duplicates were carried out using Picard (http://picard.sourceforge.net). Misalignments caused by INDELs were corrected by local re-alignment using
Genome Analysis Toolkit (GATK) and candidate SNPs were called using Variant Caller, a utility in GATK [41]. To filter variants and avoid false positives, candidate SNPs exhibiting any of the following characteristics were removed: (1) mapping quality score lower than 4; (2) quality less than 30; (3) less than $10 \times$ or more than $45 \times$ mapping depth.

Initially, SNPs of C1184 and C1234 relative to the reference genome were called separately. All of the identified SNP positions from both parental lines were then merged and compared to each other, and promising SNPs for this research between C1184 and $\mathrm{C} 1234$ were identified. The selected SNPs were used to develop dCAPS markers using the dCAPS Finder 2.0 program (http://helix.wustl.edu/dcaps) for design of nearly-matched primers including SNP positions. After designing such mismatched primers for each SNP, the opposite primers were designed using the Primer3 program (http://primer3.wi.mit. $\mathrm{edu} /$ ). All primers were synthesized by Macrogen (Seoul, Korea).

\section{Molecular marker analysis}

The newly developed dCAPS markers were validated by examining polymorphisms between the two parental lines C1184 and C1234. Additional expressed sequence tag (EST)-based dCAPS, intron-based polymorphic (IBP), genomic SSR, and INDEL markers that were not included in the previous genetic map [21] were also analyzed in this study. Furthermore, five polymorphic markers based on miniature inverted transposable element (MITE) insertion polymorphism (MIP) [42,43] were also used for genotyping the $\mathrm{F}_{2}$ population.

PCR amplifications were performed in a total volume of $25 \mu \mathrm{L}$ containing $20 \mathrm{ng}$ genomic DNA template, $1 \times$ PCR buffer, $20 \mathrm{pM}$ each primer set, $0.2 \mathrm{mM}$ each $\mathrm{dNTP}$, 1 U Taq DNA polymerase (VIVAGEN, Korea). The amplicons of dCAPS markers were mixed with $3 \mathrm{U}$

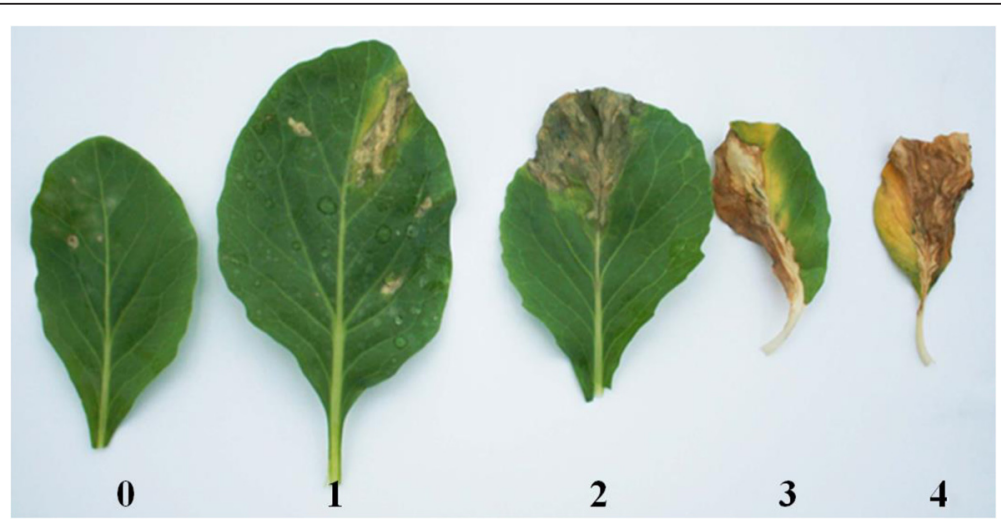

Figure 4 Representative black rot disease symptoms on leaves of $B$. oleracea after spraying with Xcc suspension. Disease indices are: (0) less than 15\%, (1) $15-30 \%$, (2) 30-55\%, (3) 55-75\%, (4) more than 75\% leaf area showing black rot symptoms. 
appropriate restriction enzymes (New England Biolabs, USA), the corresponding $1 \times$ buffer, and $1 \times$ BSA when necessary, then incubated at $37^{\circ} \mathrm{C}$ for more than three hours. The digested fragments of dCAPS markers and amplicons of other markers stained by ethidium bromide were visualized on a UV trans-illuminator after electrophoresis using 9\% non-denaturing polyacrylamide gels or $1 \%$ agarose gels depending on fragment size.

\section{Inoculation test}

Xanthomonas campestris pv. Campestris KACC 10366, obtained from the Korean Agricultural Culture Collection (KACC; Suwon, Korea) were used for the inoculation tests. Inoculum of the bacterium was scraped and cultured on tryptic soy agar (TSA) plates at $30^{\circ} \mathrm{C}$ for $48 \mathrm{~h}$. Cultured bacteria were harvested using a spreader and diluted with distilled water to $0.125 \mathrm{OD}$ at $600 \mathrm{~nm}$ to prepare bacterial suspension for inoculation.

Inoculation tests, carried out in 2012, 2013, and 2014 under the same conditions at the Korea Research Institute of Chemical Technology (Dae-jeon, Korea), were performed with $10 \sim 15 \mathrm{~F}_{3}$ plants of each individual $\mathrm{F}_{2}$ plants selected for genotyping analysis. The $\mathrm{F}_{3}$ seeds were sown and grown on $5 \times 8$ plastic pots for $20 \mathrm{~d}$ in a greenhouse. Afterwards, 20-d-old plants, usually at a stage with two sufficiently developed true leaves, were inoculated by spraying bacterial suspension until adaxial and abaxial surfaces of leaves were sufficiently wet. Each plastic pot (40 plants) received $80 \mathrm{~mL}$ bacterial suspension, and the inoculated plants were moved into a dew chamber with the temperature set at $28^{\circ} \mathrm{C}$. After $48 \mathrm{~h}$ incubation, all plants were transferred to a room maintained at $25^{\circ} \mathrm{C}$ and $80 \%$ humidity for further $7 \mathrm{~d}$ incubation with $12 \mathrm{~h}$ light/day, and disease symptoms on two inoculated leaves per each plant were surveyed. The severity of the black rot symptoms were recorded based on infected leaf area, with the following disease indices: (0) less than $15 \%$, (1) $15-30 \%$, (2) $30-55 \%$, (3) $55-75 \%$, (4) more than $75 \%$ leaf area showing black rot symptoms (Figure 4).

\section{Map construction and QTL analysis}

A total of 103 polymorphic markers were genotyped in the $F_{2}$ population, and the resulting scores were integrated into genotyping data used for a previous genetic map [21]. Linkage analysis and map construction were performed using JoinMap version 4.1 with the same parameters as in the previous study [21]. The Kosambi mapping function was used to convert recombination frequencies into genetic distances.

A disease index for each $F_{2}$ individual was calculated as the mean grade of $10 \sim 15 \mathrm{~F}_{3}$ seedlings. QTLs for Xcc resistance were evaluated using composite interval mapping (CIM) analysis with QGene program. CIM was performed with LOD (logarithm of odds) threshold values that were estimated using 1,000 permutation tests at $5 \%$ significance with 0.5 -cM scan intervals.

\section{Additional files}

\author{
Additional file 1: Table S1. Description of polymorphic markers \\ between C1184 and C1234 used in this study.
}

Additional file 2: Table S2. Results of the chi-square goodness-of-fit tests for the observed segregation ratios with the genotyped markers among $F_{2}$ plants.

Additional file 3: Figure S1. Disease index distribution of $F_{2}$ population, evaluated by average scores from inoculated $F_{3}$ plants.

\begin{abstract}
Abbreviations
QTL: Quantitative trait loci; SNP: Single nucleotide polymorphisms; Xcc: Xanthomonas campestris pv. campestris; SSR: Simple sequence repeat; NGS: Next-generation sequencing; NBS: Nucleotide binding sites; LRRs: Leucine-rich repeats; GATK: Genome analysis toolkit; EST: Expressed sequence tag; dCAPS: Derived cleaved amplified polymorphic sequences; IBP: Intron-based polymorphic; MITE: Miniature inverted transposable element; MIP: MITE insertion polymorphism; CIM: Composite interval mapping; MAS: Marker-assisted selection; CTAB: Cetyltrimethylammonium bromide.
\end{abstract}

\section{Competing interests}

The authors declare that they have no competing interests.

\section{Authors' contributions}

$H J L, N K I, H J J$, and $J$ carried out the molecular experiments. MJ and SP carried out SNP discovery. JS, JY, YS, and KA developed the mapping population and maintained plant materials. JHL and GJC performed inoculation tests and investigated disease symptoms. K-WY, JYP, and S-CL collected plant materials, and provided technical assistance. JL interpreted the results, and wrote the manuscript. YY, HK, I-SN, and T-JY conceived of and managed the research. All authors critically read and approved the final version of the manuscript.

\section{Acknowledgements}

This research was supported by the Golden Seed Project (Center for Horticultural Seed Development, No. 213003-04-1-SB430), Ministry of Agriculture, Food and Rural Affairs (MAFRA), Ministry of Oceans and Fisheries (MOF), Rural Development Administration (RDA) and Korea Forest Service (KFS).

\section{Author details}

${ }^{1}$ Department of Plant Science, Plant Genomics and Breeding Institute, and Research Institute of Agriculture and Life Sciences, College of Agriculture and Life Sciences, Seoul National University, Seoul 151-921, Republic of Korea. ${ }^{2}$ Indonesian Research Institute for Industrial and Beverage Crops (IRIIBC), Pakuwon, Sukabumi, Indonesia. ${ }^{3}$ Department of Horticulture, Sunchon National University, Suncheon 540-950, Republic of Korea. ${ }^{4}$ Joeun Seed, \#174, Munbang-Ri, Cheonhan-Myun, 367-833 Goesan-Gu, Chungcheongbuk-Do, Korea. ${ }^{5}$ Research Center for Biobased Chemistry, Korea Research Institute of Chemical Technology, Daejeon 305-600Yusong-Gu, Republic of Korea. ${ }^{6}$ Arizona Genomics Institute, School of Plant Sciences, University of Arizona, Tucson, Arizona 85721, USA. ${ }^{7}$ Department of Agricultural Biotechnology, Seoul National University, Seoul 151-921, Republic of Korea. ${ }^{8} \mathrm{CHO} \& \mathrm{KIM}$ genomics, Seoul National University Mt.4-2, Main Bldg. \#514, SNU Research Park, NakSeoungDae, Seoul 151-919Gwanakgu, Republic of Korea.

Received: 25 September 2014 Accepted: 15 January 2015 Published online: 03 February 2015

\section{References}

1. UN. Genome analysis in Brassica with special reference to the experimental formation of $B$. napus and peculiar mode of fertilization. J Japanese Bot. 1935;7:389-452. 
2. Liu S, Liu Y, Yang X, Tong C, Edwards D, Parkin IA, et al. The Brassica oleracea genome reveals the asymmetrical evolution of polyploid genomes. Nature Commun. 2014;5:3930.

3. Parkin IA, Koh C, Tang H, Robinson SJ, Kagale S, Clarke WE, et al. Transcriptome and methylome profiling reveals relics of genome dominance in the mesopolyploid Brassica oleracea. Genome Biol. 2014;15(6):R77.

4. Kifuji Y, Hanzawa H, Terasawa Y, Nishio T. QTL analysis of black rot resistance in cabbage using newly developed EST-SNP markers. Euphytica. 2013;190(2):289-95.

5. Tonu NN, Doullah MA-u, Shimizu M, Karim MM, Kawanabe T, Fujimoto R, et al. Comparison of positions of QTLs conferring resistance to xanthomonas campestris pv. campestris in Brassica oleracea. Am J Plant Sci. 2013;4:11.

6. Williams P. Black rot: a continuing threat to world crucifers. Plant Dis. 1980;64(8):736-42.

7. Jensen BD, Massomo SM, Swai IS, Hockenhull J, Andersen SB. Field evaluation for resistance to the black rot pathogen Xanthomonas campestris pv. campestris in cabbage (Brassica oleracea). Eur J Plant Pathol. 2005; 113(3):297-308.

8. Hutton SF, Scott JW, Yang W, Sim S-C, Francis DM, Jones JB. Identification of QTL associated with resistance to bacterial spot race T4 in tomato. Theor Appl Genet. 2010;121(7):1275-87.

9. Fargier E, Manceau C. Pathogenicity assays restrict the species Xanthomonas campestris into three pathovars and reveal nine races within $X$. campestris pv. campestris. Plant Pathol. 2007;56(5):805-18.

10. Vicente J, Conway J, Roberts S, Taylor J. Identification and origin of Xanthomonas campestris pv. campestris races and related pathovars. Phytopathology. 2001;91(5):492-9.

11. Soengas P, Hand P, Vicente J, Pole J, Pink D. Identification of quantitative trait loci for resistance to Xanthomonas campestris pv. campestris in Brassica rapa. Theor Appl Genet. 2007;114(4):637-45.

12. Duran C, Appleby N, Edwards D, Batley J. Molecular genetic markers: discovery, applications, data storage and visualisation. Curr Bioinformatics. 2009;4(1):16-27.

13. Hendre PS, Aggarwal RK. DNA markers: development and application for genetic improvement of coffee. In: Varshney RK, Tuberosa R, editors. Genomics-assisted crop improvement. 2nd ed. Netherlands: Springer; 2007. p. 399-434.

14. Tanksley SD, McCouch SR. Seed banks and molecular maps: unlocking genetic potential from the wild. Science. 1997;277(5329):1063-6.

15. Camargo L, Williams $P$, Osborn T. Mapping of quantitative trait loci controlling resistance of Brassica oleracea to Xanthomonas campestris pv. campestris in the field and greenhouse. Phytopathology. 1995;85(10):1296-300.

16. Doullah M, Mohsin G, Ishikawa K, Hori H, Okazaki K. Construction of a Linkage Map and QTL analysis for Black Rot Resistance in Brassica oleracea L. Int J Nat Sci. 2011;1(1):1-6.

17. Yu Z, Guo X. Identification and mapping of disease-resistance QTLs in the eastern oyster, Crassostrea virginica Gmelin. Aquaculture. 2006;254(1):160-70.

18. Mammadov J, Aggarwal R, Buyyarapu R, Kumpatla S. SNP markers and their impact on plant breeding. Int J Plant Genomics. 2012;2012:11.

19. Rafalski JA. Novel genetic mapping tools in plants: SNPs and LD-based approaches. Plant Sci. 2002;162(3):329-33.

20. Wang W, Huang S, Liu Y, Fang Z, Yang L, Hua W, et al. Construction and analysis of a high-density genetic linkage map in cabbage (Brassica oleracea L. var. capitata). BMC Genomics. 2012;13(1):523.

21. Izzah NK, Lee J, Jayakodi M, Perumal S, Jin M, Park B-S, et al. Transcriptome sequencing of two parental lines of cabbage (Brassica oleracea L. var. capitata L.) and construction of an EST-based genetic map. BMC Genomics. 2014;15(1):149

22. Hammond-Kosack KE, Jones JD. Plant disease resistance genes. Annu Rev Plant Biol. 1997:48(1):575-607

23. Cheng F, Liu S, Wu J, Fang L, Sun S, Liu B, et al. BRAD, the genetics and genomics database for Brassica plants. BMC Plant Biol. 2011;11(1):136.

24. Bentley DR. Whole-genome re-sequencing. Curr Opin Genet Dev. 2006;16(6):545-52

25. Liu J, Huang S, Sun M, Liu S, Liu Y, Wang W, et al. An improved allele-specific PCR primer design method for SNP marker analysis and its application. Plant Methods. 2012;8(1):34.

26. Lysak MA, Koch MA, Pecinka A, Schubert I. Chromosome triplication found across the tribe Brassiceae. Genome Res. 2005;15(4):516-25.
27. Yang T-J, Kim JS, Kwon S-J, Lim K-B, Choi B-S, Kim J-A, et al. Sequence-level analysis of the diploidization process in the triplicated flowering locus $C$ region of Brassica rapa. Plant Cell Online. 2006;18(6):1339-47.

28. Wang $X$, Wang H, Wang J, Sun R, Wu J, Liu S, et al. The genome of the mesopolyploid crop species Brassica rapa. Nat Genet. 2011;43(10):1035-9.

29. Iniguez-Luy FL, Lukens L, Farnham MW, Amasino RM, Osborn TC. Development of public immortal mapping populations, molecular markers and linkage maps for rapid cycling Brassica rapa and B. oleracea. Theor Appl Genet. 2009;120(1):31-43.

30. Alheit KV, Reif JC, Maurer HP, Hahn V, Weissmann EA, Miedaner T, et al. Detection of segregation distortion loci in triticale (x Triticosecale Wittmack) based on a high-density DArT marker consensus genetic linkage map. BMC Genomics. 2011;12(1):380.

31. Taylor J, Conway J, Roberts S, Astley D, Vicente J. Sources and origin of resistance to Xanthomonas campestris pv. campestris in Brassica genomes. Phytopathology. 2002;92(1):105-11.

32. Vicente J, Taylor J, Sharpe A, Parkin I, Lydiate D, King G. Inheritance of race-specific resistance to Xanthomonas campestris $\mathrm{pv}$. campestris in Brassica genomes. Phytopathology. 2002;92(10):1134-41.

33. Schranz ME, Lysak MA, Mitchell-Olds T. The ABC's of comparative genomics in the Brassicaceae: building blocks of crucifer genomes. Trends Plant Sci. 2006;11(11):535-42.

34. Holub EB. The arms race is ancient history in Arabidopsis, the wildflower. Nat Rev Genet. 2001;2(7):516-27.

35. Leister D. Tandem and segmental gene duplication and recombination in the evolution of plant disease resistance genes. Trends Genet. 2004;20(3):116-22.

36. Town CD, Cheung F, Maiti R, Crabtree J, Haas BJ, Wortman JR, et al. Comparative genomics of Brassica oleracea and Arabidopsis thaliana reveal gene loss, fragmentation, and dispersal after polyploidy. Plant Cell. 2006;18(6):1348-59.

37. Meyers BC, Kozik A, Griego A, Kuang H, Michelmore RW. Genome-wide analysis of NBS-LRR-encoding genes in Arabidopsis. Plant Cell. 2003;15(4):809-34.

38. Allen G, Flores-Vergara M, Krasynanski S, Kumar S, Thompson W. A modified protocol for rapid DNA isolation from plant tissues using cetyltrimethylammonium bromide. Nat Protoc. 2006;1(5):2320-5.

39. DePristo MA, Banks E, Poplin R, Garimella KV, Maguire JR, Hartl C, et al. A framework for variation discovery and genotyping using next-generation DNA sequencing data. Nat Genet. 2011:43(5):491-8.

40. Langmead B, Salzberg SL. Fast gapped-read alignment with Bowtie 2 . Nat Methods. 2012;9(4):357-9.

41. Nekrutenko A, Taylor J. Next-generation sequencing data interpretation: enhancing reproducibility and accessibility. Nat Rev Genet. 2012;13(9):667-72.

42. Sampath $P$, Lee S-C, Lee J, Izzah NK, Choi B-S, Jin M, et al. Characterization of a new high copy stowaway family MITE, BRAMI-1 in Brassica genome. BMC Plant Biol. 2013;13(1):56

43. Sampath P, Murukarthick J, Izzah NK, Lee J, Choi H-I, Shirasawa K, et al. Genome-wide comparative analysis of 20 miniature inverted-repeat transposable element families in Brassica rapa and B. oleracea. PLoS One. 2014;9(4):e94499.

\section{Submit your next manuscript to BioMed Central and take full advantage of:}

- Convenient online submission

- Thorough peer review

- No space constraints or color figure charges

- Immediate publication on acceptance

- Inclusion in PubMed, CAS, Scopus and Google Scholar

- Research which is freely available for redistribution 